\title{
Assessing the Emotional Experience of Soap Bubbles as Input Material for Interactive Games
}

\author{
Tanja Döring, Franziska Lorz, Rainer Malaka \\ AG Digitale Medien, Universität Bremen
}

\begin{abstract}
In this paper, we assess users' emotional experiences of real soap bubbles as input material in an interactive game. We designed a game that can be controlled via soap bubbles. In a user study we compared playing the game with bubbles versus with transparent plastic spheres as handles for input. We collected data on users' emotions and user experience by using the PANAS-X and the User Experience Questionnaire as well as by observing the participants during the interaction sessions. The study shows that the interaction with real bubbles is more playful and causes more surprise when used for the first time in a digital game.
\end{abstract}

\section{Introduction}

How do the material properties of interaction elements influence the users' experience and the users' emotions? This is, in general, a question that designers of tangible user interfaces (TUIs) should address. It is, of course, a question that cannot be answered generically, but depends on the specific setting, the users and their previous experiences and values, the application context, the way the material is physically embedded and so on. Research in TUI design has not put much effort in systematically exploring this design space to foster a deeper understanding about material aspects for interaction. Often, physical materials can be integrated to very different degrees into a user interface: e.g., as a digital representation, as a metaphorical physical object, or as the real material itself.

In this work, we focus on the integration of soap bubbles as an unusual, engaging and ephemeral material into applications (for related work on ephemeral user interfaces see Döring et al. 2013). From digital representations of bubbles (e.g., Okuno et al. 2003) to metaphorical bubble objects (e.g., Bernhaupt et al. 2014) to real bubbles (e.g., Nakamura et al. 2006) some applications and installations exist already that exemplarily explore this design 
space. Yet, it has not been evaluated how these different representations of bubbles affect the users' emotional experience of a user interface. We address this question and present a study, in which we compare real soap bubbles and transparent plastic spheres (plastic "bubbles") as input material in an interactive game. We collected data on users' emotions and user experience (UX) by using the PANAS-X and the User Experience Questionnaire (UEQ) as well as by observing the participants during the sessions. This work builds upon previous work on soap bubbles for interaction (e.g. Döring et al. 2012). The contributions of this paper contain the presentation of an interactive game with soap bubble interface as well as a user study comparing real soap bubbles and plastic "bubbles" as game user interfaces.

\section{Soap Bubbles as Interaction Material}

Soap bubbles have been a topic of interest in science, art, and literature for many years (c.f. Emmer 1987). One of the major connotations soap bubbles evoke in grown-ups and kids is playful interaction. Furthermore, bubbles have a number of meanings: they are used as symbols for vanity in art and in a number of metaphorical expressions stressing short life spans or lacking in content. They form shapes that build "in-between" spaces-spaces that are neither real nor fully virtual (Clausen 2002) and generally evoke fascination. In the context of user interfaces, they have been addressed recently as interesting material for interaction. While most examples use bubbles as output media, we are especially interested in using them as input material in order to explore how the material with its special properties and meanings shape interactions.

\section{The Game "Bubblesearch"}

In order to examine real soap bubbles as input means in an interactive game, we combined a soap bubble interface (Sylvester et al. 2010) with a screen visualizing an underwater game environment. The basic setup of the soap bubble interface can be seen in Figure 1. It consists of a housing with bubble generator and a basin with transparent acrylic glass surface, on top a thin layer of colored dark liquid. The basin has a diameter of approx. $50 \mathrm{~cm}$. By pressing a button on a control panel soap bubbles can be generated that drop onto the basin surface, where they float as half spheres in the liquid surface until they break. Beneath the surface is a camera that tracks the floating bubbles so that their locations can be used in an application. In order to move the bubbles, a variety of different interaction techniques are possible, e.g. touching and moving bubbles with wet fingers or blowing. Of course, they burst when touched too ungently or by themselves after a while, which is part of the fun when playing the game.

In our game "Bubblesearch", we used the bubbles to control circle-shaped peepholes (they could also represent see-through air bubbles) in an underwater world that is covered with a black layer (see Figure 2). The screen with visual output was projected to the wall behind the soap bubble interface. We realized this indirect interaction setting in order to provide visuals 


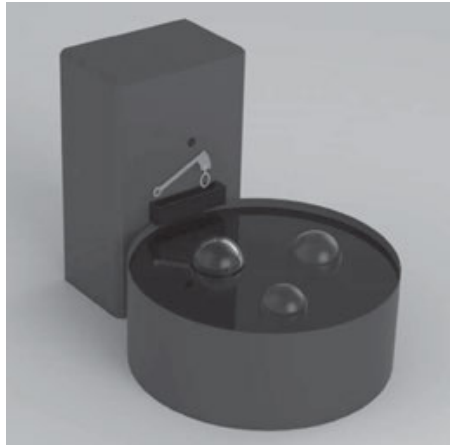

Figure 1. The Soap Bubble Interface with bubble generator and soap bubble basin. The basin has a diameter of approx. $50 \mathrm{~cm}$. Generated bubbles drop onto the liquid surface where they float.

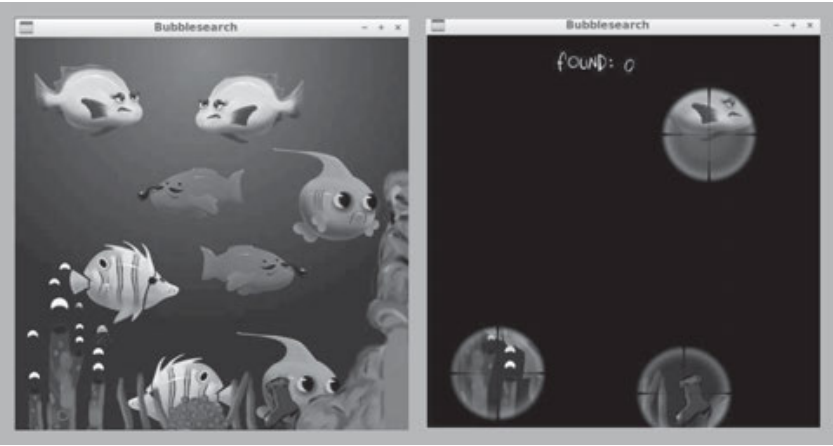

Figure 2. Graphical Output of the Game "Bubblesearch". Left: Underwater world with pairs of matching fishes. Right: View as in the game (projected onto the wall behind the soap bubble interface). The fishes are hidden beneath a black layer and need to be uncovered by moving soap bubbles to the according locations.

with a constant good quality in both conditions in our comparative user study. While a projection directly onto the liquid surface would be desirable for the setup in future, a visual output in the basin and on the bubbles with a sufficient good quality to play the game in both versions was still challenging to realize. The goal of the game was to find pairs of matching fishes, which moved around horizontally in the underwater world. In order to detect a pair it was necessary to have two peepholes on top of each of the fishes that belonged to a pair at the same time. As the peepholes were tied to the referring location of the soap bubbles in the basin, the bubbles had to be moved to the spots were the two fishes of one pair were expected by the player. Whenever parts of both fishes were visible in the peepholes, a pair was directly counted as detected and the score was displayed on the screen. We tracked four soap bubbles at maximum.

\section{User Study}

We conducted a user study, in which we compared the above-described setting (using real soap bubbles as input handles) to a setting with transparent plastic half spheres (see Figure 3). The plastic half spheres, which had about the same size as the generated bubbles, rested next to the basin and needed to be placed on the liquid surface in order to use them. The same circle-based tracking was used as with the real bubbles. The plastic half spheres were also linked in the same manner to the peepholes as the bubbles. Both interface materials differ in a variety of appearances and handlings. E.g. the bubbles can be generated but they break, they deform, and they are hard to control. The plastic half spheres last, are stable in their shape and can be moved much easier and faster. We were interested in investigating how the emotional response and the UX of the players differed between both interface instances. We collected quantitative data from questionnaires as well as qualitative data from videotaping, observation and short interviews at the end of the session. Emotional response was measured with selected modules of the PANAS-X questionnaire (Watson and 


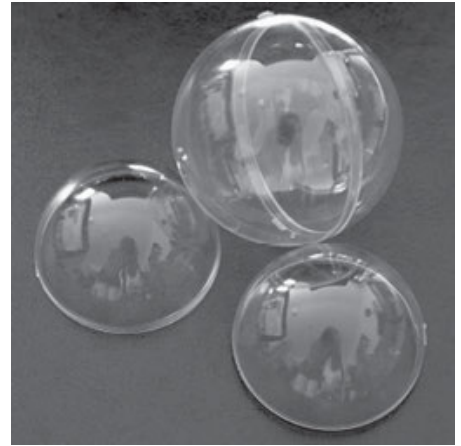

Figure 3. Transparent plastic spheres as Figure 4. The study setup. The indirect interaction took place alternative input handles.

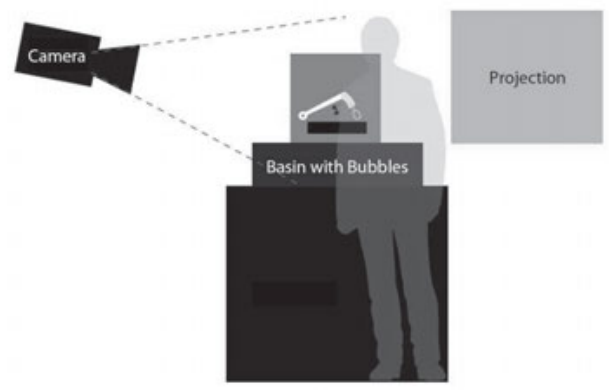

via moving real bubbles or plastic half spheres on the liquid surface of the basin while the game screen was displayed at the wall behind the soap bubble machine.

Clark 1994). Next to the basic modules assessing positive and negative affect we used the modules for the affective states fatigue, surprise and serenity. Our PANAS-X questionnaire contained 36 attributes that describe different feelings and emotions. Users rated their agreement with the corresponding emotion on a 1-5 point scale from "not at all" to "extremely". UX was assessed with the UEQ questionnaire (Laugwitz et al. 2008). It collects data about the users' assessment of the attractiveness, stimulation, novelty, efficiency, perspicuity, and dependability of the system. The questionnaire contains 26 pairs of opposite attributes that are assessed on a 1-7 point scale.

The general setup can be seen in Figure 4. We conducted a within group design lab-based study, in which 10 players used both interfaces, half of them first with soap bubbles and the other half first with plastic half spheres, one player at a time. The overall procedure was as follows. First, a new participant had to fill out the PANAS-X questionnaire in order to gather his or her mood before the experiment. Then, the first round of playing the game took place with either of the two input conditions. The experimenter briefly explained the game, but did not give hints how to use the interface components in order to see what strategies the players developed. The participant played the game for five minutes. Then it was interrupted and he or she filled in another PANAS-X as well as a UEQ questionnaire. After that, a second round of the game was played with the other interface condition for five minutes, again, followed by a PANAS-X and a UEQ assessment. In the end, the experimenter asked the player which interface condition he or she preferred. Overall, a session lasted approximately 30 minutes. The found pairs of fishes were counted, and all sessions were videotaped.

\section{Results}

Five females and five males with an age range from 22 to 54 years participated in the study. None had seen the soap bubble interface before. All participants played the game in both conditions (soap bubbles: SB, plastic half spheres: PS). The average pairs of fishes found differed largely between both conditions: while in the plastic sphere condition on average 


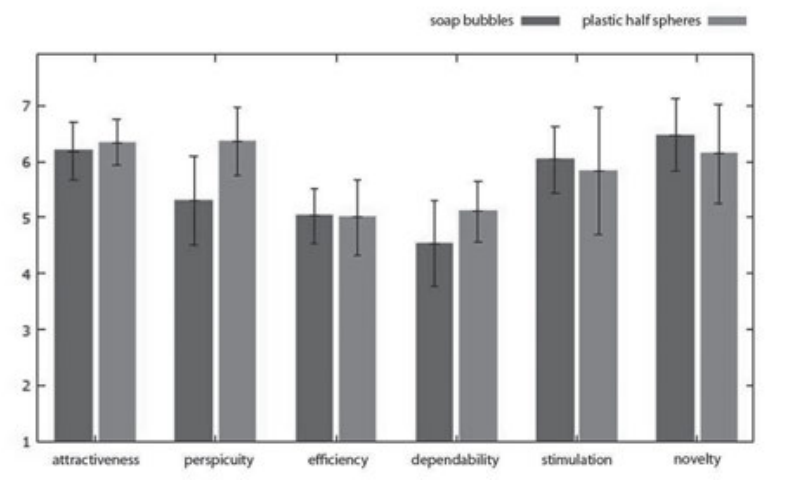

Figure 5. Results of the User Experience Questionnaire

(7 point scale, error bars indicate standard deviation).

10.5 pairs could be found, in the soap bubble condition only 2.8 pairs were discovered. This first of all shows that the game is much more challenging to play with soap bubbles due to their fragility and limited controllability. But it also reveals that the game as we designed it was generally playable with the bubbles. In our observations it was striking how much more the soap bubbles triggered a playful interaction. Many participants played around with them, tried out different interaction techniques and explored the underwater world beyond the goal of the game. In order to move the soap bubbles, the participants developed very diverse, novel and creative techniques, i.e. blowing, waving air, touching and moving bubbles gently with one finger, grasping and positioning them with the full hand (with five wet fingers), or moving the surrounding liquid with the flat of the hand into the desired direction. This diversity stands in contrast to the handling of the plastic bubbles, which were generally simply grasped with the thumb and one or more fingers of one hand.

The results of the UEQ questionnaire (7 point scale) revealed that the players generally gave high scores in all dimensions of assessed UX for both conditions (see Figure 5). Especially regarding attractiveness and design quality (measured through novelty and stimulation), both interfaces, soap bubbles and plastic spheres, received very high scores. Nevertheless, it surprises that both interaction styles evoked a similar impression regarding efficiency (SB: $\mathrm{M}=5.03, \mathrm{SD}=0.49$; $\mathrm{PS}: \mathrm{M}=5.00, \mathrm{SD}=0.66$; scale $1-7$ ) although the players generally achieved a higher score in the game with the plastic bubbles. The biggest difference in the assessment of the user interface versions was found regarding perspicuity with better results for the PS condition ( $\mathrm{SB}: \mathrm{M}=5.31, \mathrm{SD}=0.80$; $\mathrm{PS}: \mathrm{M}=6.36, \mathrm{SD}=0.61$; scale 1-7). This mainly shows that users found the interaction with the plastic half spheres simpler and easier to learn, which is not surprising regarding the higher challenge when interacting with the soap bubbles. With a mean value of $4.53(\mathrm{SD}=0.76)$ dependability in the soap bubble condition received the lowest score, which is very likely directly influenced by the degree of unpredictability of the interaction material itself. Overall, the UEQ results revealed that the perceived user experience of both UI versions was generally high. Although the game was more challenging in the soap bubble condition, this does not negatively affect the overall user experience. The game setup was experienced as novel and innovative in both interface conditions. 
With the PANAS-X questionnaire we measured positive affect, negative affect, fatigue, surprise and serenity at the beginning of the study, after trial 1 and at the end after trial 2 on a five point scale $(1=$ not at all to $5=$ =xtremely). Figure 6 a shows the average scores by the participants regarding the surveyed factors. Regarding negative affect (dark blue line), we see an on average very low affect between 1 and 1.5 at the beginning of the study that does not get higher after interacting with the game. While Figure 6a shows both conditions together, also the separate examination of the conditions reveals that the negative affect stays constantly low (see Figure 6b). The positive affect (orange line) however initially is on average between 3 and 3.5 and slightly rises after interacting with the game (before trial 1: $\mathrm{M}=3.31, \mathrm{SD}=0.61$, after trial 2: $\mathrm{M}=3.79, \mathrm{SD}=0.43$; scale $1-5$ ). When comparing users who interacted with the soap bubbles first (SB first) to users who interacted with the plastic spheres first (PS first), we find a stronger increase for the group SB first. Fatigue (blue line) overall is initially relatively low and on average gets a little lower during the game play (before trial 1: $\mathrm{M}=1.90, \mathrm{SD}=0.60$, after trial 2: $\mathrm{M}=1.50, \mathrm{SD}=0.66$; scale $1-5)$. The scores for serenity dropped a little during the first trial and have risen again after the second trial, no matter which interface condition was first (see Figure 6b). This might indicate that the users were a little less relaxed after the first use of the interface due to the novelty but felt a little more relaxed again after round 2 already when they got more familiar with the game. Finally, the affect surprise rose strikingly during the first game trial (green line, before trial 1: $\mathrm{M}=2.03, \mathrm{SD}=1.07$, after trial $1: \mathrm{M}=3.30, \mathrm{SD}=1.16$; scale $1-5$ ). When we look separately at groups SB first and PS first, we find that especially the SB first group was strongly affected by surprise (before trial 1: $\mathrm{M}=1.60, \mathrm{SD}=0.98$, after trial 1: $\mathrm{M}=3.87, \mathrm{SD}=0.56$; scale 1-5). This means that, especially for first time users, using soap bubbles as input means for interactive applications can evoke a high emotional affect regarding surprise. In general, the users liked both UI variations. Four users preferred the soap bubbles, finding them challenging, fun and exciting. Three preferred the plastic bubbles arguing that they are easier to use and thus more motivating, and the three remaining players had no preference among the two interface instances.

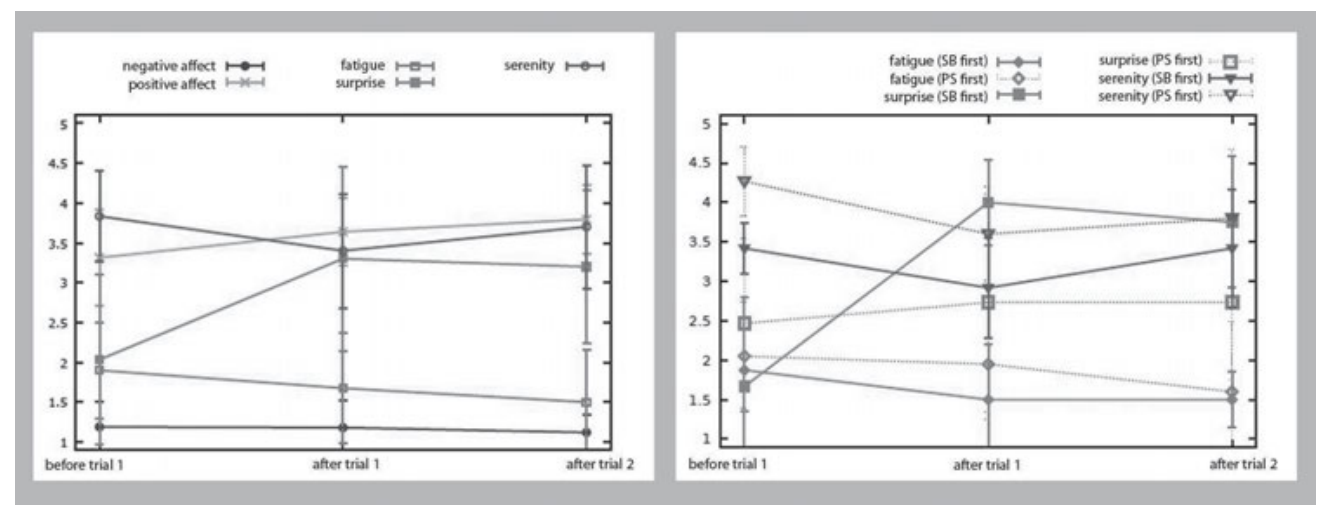

Figure 6. Results of the PANAS-X questionnaire (5 point scale, error bars indicate standard deviation). Left (6a): Mean values of all dimensions for all users. Right (6b): Mean values for fatigue, surprise and serenity, separately of users who used the real soap bubbles first (SB) and of users who used the plastic spheres first (PS). 


\section{Conclusion}

We presented a study in which we assessed the emotional experience of soap bubbles versus plastic spheres as input means for an interactive game. We found that real bubbles foster playful and diverse interactions to a greater extent than plastic bubbles do. Although the game was more challenging to play with the real bubbles, the perceived UX was equally high as in the plastic bubble condition. Similarly, both interfaces evoked a slightly growing positive affect and a constantly low negative affect. Regarding surprise, the real soap bubbles provoked a high affect. Overall, our results indicate that both bubble input versions are suited as input means in interactive games. The real bubbles especially seem to fit application contexts, where users' attention needs to be raised, and where they should be animated for playful interaction.

\section{Acknowledgements}

We thank Marc Herrlich for his advice on planning the evaluation and Daniel Vidal Moya for realizing the graphics for the fishes.

\section{References}

Bernhaupt, R., Pirker, M., \& Desnos, A. (2014). The Bubble User Interface: A Tangible Representation of Information to Enhance the User Experience in IPTV Systems, in: Proc. of DIS Companion '14. ACM, pp. 85-88.

Clausen, B (2002). Thin Skin. The Fickle Nature of Bubbles, Spheres, and Inflatable Structures. New York : Independent Curators International.

Döring, T., Sylvester, A., \& Schmidt, A (2012). Exploring material-centered design concepts for tangible interaction, in: Proc. of CHI Extended Abstracts 2012, ACM, pp. 1532-1528.

Döring, T., Sylvester, A., \& Schmidt, A (2013). A Design Space for Ephemeral User Interfaces, in: Proc. of TEI'13. ACM, pp. 75-82.

Emmer, M. (1987). Soap Bubbles in Art and Science: From the Past to the Future of Math Art. In: Leonardo, Vol. 20, No. 4, MIT Press, pp. 327-334.

Laugwitz, B., Schrepp, M., \& Held, T (2008). Construction and evaluation of a user experience questionnaire. In: Holzinger, A. (Ed.): USAB 2008, LNCS 5298, pp. 63-76.

Nakamura, M., Inaba, G., Tamaoki, J., Shiratori, K., \& Hoshino, J. (2006). Bubble Cosmos, in: ACM SIGGRAPH 2006 Emerging Technologies, SIGGRAPH '06. ACM, Article 3.

Okuno, Y., Kakuta, H., Takayama, T., \& Asai, K. (2003). Jellyfish Party: Blowing Soap Bubbles in Mixed Reality Space, in: Proc. of ISMAR '03. IEEE, pp. 358-359.

Sylvester, A., Döring, T., \& Schmidt, A. (2010). Liquids, smoke, and soap bubbles: reflections on materials for ephemeral user interfaces, in: Proc. of TEI '10. ACM, pp. 269-270.

Watson, D. \& Clark, L. A. (1994). The PANAS-X: Manual for the Positive and Negative Affect Schedule - expanded Form / The University of Iowa. 1994. 


\section{Kontaktinformationen}

Tanja Döring, Franziska Lorz, Rainer Malaka

AG Digitale Medien

Universität Bremen

Bibliothekstr. 1

28359 Bremen, Deutschland 